\title{
ON THE FOUNDATIONS OF THE CALCUL FONCTIONNEL OF
}

\section{FRÉCHET*}

BY

\section{A. D. PITCHER AND E. W. CHITTENDEN}

In his thesis, $\uparrow$ Fréchet gave a very beautiful generalization of the theory of point sets and of the theory of real valued functions of a real variable. His functions are real valued but the range of the independent variable is an abstract class $\mathfrak{O}$ of elements $q$. He secures his results principally through the medium of a properly conditioned distance function $\delta$, a generalization of the distance between two points, which associates with each pair $q_{1} q_{2}$ of elements a real non-negative number $\delta\left(q_{1} q_{2}\right)$. He is thus enabled to secure the more important theorems of point set theory and of real function theory, especially those relating to continuous functions and their properties. This theory of Fréchet has excited considerable interest and has received much attention from mathematicians. Various contributions to its foundations and to its content have been made.

In the present paper we follow the example of Fréchet in assuming once for all that $\delta(q q)=0$ and that $\delta\left(q_{1} q_{2}\right)=\delta\left(q_{2} q_{1}\right)$. In other words we assume that the distance from an element to itself is zero and that the distance between two elements is independent of the order in which they are taken. In the first part of the paper we give very simple conditions on systems $(\mathfrak{Q} ; \delta)$ which are sufficient for many purposes and which, in the case of compact sets, we show to be equivalent, so far as limit of a sequence is concerned, to the voisinage and thus to the écart of Fréchet. $\ddagger$ The remainder of the paper is devoted to the theory of functions on the sets $\mathfrak{Q}$ of systems $(\mathfrak{D} ; \delta)$. In terms of the conditions already mentioned we generalize the results of Fréchet and Hahn as to the existence of non-constant continuous functions. We give two very

* The results of this paper have been presented to the Society at various times, especially April 2, 1915, and December 28, 1916.

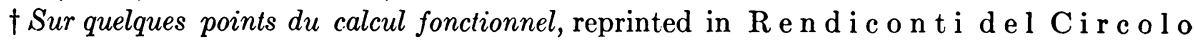
M a t e m a t i c o d i Pa le r m o, vol. 22 (1906), pp. 1-74.

See also, $A$ contribution to the foundations of Fréchet's calcul fonctionnel, by T. H. Hildebrandt, American Journal of Mathematics, vol. 34 (1912), pp. 237-290.

$\ddagger \mathrm{E}$. W. Chittenden has proved that the voisinage and the écart of Fréchet are equivalent so far as the limit of a sequence is concerned. Cf. these $\mathrm{T} \mathrm{r}$ a $\mathrm{n} \mathrm{s}$ a c t i o n s, vol. 18 (1917), pp. 161-166. 
mild conditions on systems $(\mathfrak{Q} ; \delta)$ which are completely independent and which secure for all continuous functions on $\mathfrak{Q}$ the important properties of the continuous functions of a real variable. Also we give a set of three completely independent conditions (the two above and one other) which we show to be both necessary and sufficient for a so-called uniformly proper theory of continuous functions where the term uniformly proper is used in a sense likely to be admitted by any one who gives the matter careful consideration.

\section{INTRODUCTION}

By the notation $(\mathfrak{Q} ; \delta$ ) we denote a set $\mathfrak{Q}$ of elements $q$ and a function $\delta$ which assigns to each pair $q_{1} q_{2}$ of elements a real number $\delta\left(q_{1} q_{2}\right) \geqq 0$. We also assume, once for all, that for every element $q, \delta(q q)=0$, and that for every pair $q_{1}, q_{2}$ of elements $\delta\left(q_{1} q_{2}\right)=\delta\left(q_{2} q_{1}\right)$. We follow Fréchet in saying that $q$ is a limit of the sequence $q_{n}, L_{n} q_{n}=q$, when and only when $L_{n} \delta\left(q_{n} q\right)=0$. We shall be interested in the following properties of $\delta$, or of systems $(\mathfrak{Q} ; \delta)$.

(1) If $\delta\left(q_{1} q_{2}\right)=0$ then $q_{1}=q_{2}$.

(2) If $L_{n} q_{1 n}=q$ and $L_{n} \delta\left(q_{1 n} q_{2 n}\right)=0$ then $L_{n} q_{2 n}=q$.

(3) If $L_{n} q_{1 n}=q=L_{n} q_{2 n}$ then $L_{n} \delta\left(q_{1 n} q_{2 n}\right)=0$.

(4) If $L_{n} \delta\left(q_{1 n} q_{2 n}\right)=0$ and $L_{n} \delta\left(q_{2 n} q_{3 n}\right)=0$ then $L_{n} \delta\left(q_{1 n} q_{3 n}\right)=0$.

(5) There is a function $\phi(e)$ such that $L_{e=0} \phi(e)=0$ and such that if $\delta\left(q_{1} q_{2}\right) \leqq e, \delta\left(q_{2} q_{3}\right) \leqq e$ then $\delta\left(q_{1} q_{3}\right) \leqq \phi(e)$.

(6) $\delta\left(q_{1} q_{2}\right)+\delta\left(q_{2} q_{3}\right) \geqq \delta\left(q_{1} q_{3}\right)$.

It will be seen at once that (2), (3), and (4) are important properties which are implied by (5) or (6). We will show that (2), (3), and (4) play a fundamental rôle.

The notation $\delta^{n}$ denotes the fact that $\delta$ has the property $(n)$. Thus $\delta^{13}$ denotes a $\delta$ which possesses the properties (1) and (3). The voisinage of Fréchet is a $\delta^{15}$ and the écart a $\delta^{16}$.

Our terminology is that of Fréchet except that we do not wish to imply that the limit of a sequence is unique. Thus a set $\overline{\mathfrak{D}}$ of the set $\mathfrak{Q}$ of a system $(\mathfrak{Q} ; \delta)$ is said to be compact in case every sequence of distinct elements of $\overline{\mathfrak{Q}}$ gives rise to at least one limiting element. $\mathfrak{\mathfrak { D }}$ is closed in case every limiting element of $\overline{\mathfrak{D}}$ is of $\overline{\mathfrak{D}}$. It should be noted that, in a system $(\mathfrak{Q} ; \delta)$ where $\delta\left(q_{1} q_{2}\right)$ may be zero for $q_{1}$ and $q_{2}$ distinct, an element $q$ may be the limit of a sequence composed of a single element, other than $q$ itself, repeated infinitely often. If $\overline{\mathfrak{D}}$ is compact and closed then $\overline{\mathfrak{D}}$ is said to be extremal. For the case of non-unique limits it is desirable to take special note of classes $\overline{\mathfrak{D}}$ which may not be closed but which are such that every sequence $\left\{q_{n}\right\}$ of $\overline{\mathfrak{Q}}$, which has a limit, has a limit in $\overline{\mathfrak{D}}$. Such sets are said to be self-closed. If a set $\overline{\mathfrak{\Omega}}$ is compact and self-closed it is said to be self-compact. The property (2) will 
prove to be of fundamental importance and, for lack of a better term we venture to call a system $\left(\mathfrak{Q} ; \delta^{2}\right)$ a coherent $\operatorname{system} .^{*}$ A system $(\mathfrak{Q} ; \delta)$ is said to be limited if there is a positive number $h$ such that for every $q_{1} q_{2}$, $\delta\left(q_{1} q_{2}\right) \leqq h$.

We may have two systems $(\mathfrak{Q} ; \delta)$ and $(\mathfrak{Q} ; \bar{\delta})$, the set $\mathfrak{Q}$ being the same in each case but the two distance functions, $\delta$ and $\bar{\delta}$, not the same. If we wish to indicate that $q$ is a limit of the sequence $\left\{q_{n}\right\}$ we write, in the first case, $L_{n} q_{n}=q$, and in the second case, $L_{n} q_{n}=q$. Two systems $(\mathfrak{Q} ; \delta)$ and $(\mathfrak{Q} ; \bar{\delta})$ are said to be $L$-equivalent in case $L_{n} q_{n}=q$ implies $\bar{L}_{n} q_{n}=q$ and conversely.

Denote by $\mathfrak{P}$ a class of elements $p$. If $\mathfrak{P}$ is a subclass (reduction $\dagger$ ) of $\mathfrak{Q}$ of a system $(\mathfrak{Q} ; \delta)$, we denote by $(\mathfrak{B} ; \delta)$ a reduced system where $\delta\left(p_{1} p_{2}\right)$ $=\delta\left(q_{1} q_{2}\right)$ in case $p_{1}=q_{1}$ and $p_{2}=q_{2}$. It will be noted that, if a system $(\mathfrak{Q} ; \delta)$ has any one of the properties $(1) \cdots(5)$, then any reduction of $(\mathfrak{Q} ; \delta)$ has that property also.

We speak of extremal systems $(\mathfrak{D} ; \delta)$, closed systems $(\mathfrak{Q} ; \delta)$, etc., in an obvious way. A compact system is a reduction of an extremal system.

Two elements $\bar{q}, \tilde{q}$ of a set $\mathfrak{Q}$ of a system $(\mathfrak{Q} ; \delta)$ are said to be connected $\ddagger$ by $\delta$ if for every $e$ there is a finite sequence $q_{1 e}, q_{2 e}, \cdots, q_{k_{e}}$ such that $q_{1 e}=\bar{q}$, $q_{k_{\mathrm{e}} e}=\tilde{q}$ and such that $\delta\left(q_{i e} q_{i+1} e\right) \leqq e, i=1,2, \cdots, k_{e}-1$. The set $\mathfrak{Q}$ is a connected set, or the system $(\mathfrak{Q} ; \delta)$ is a connected system, in case every pair $q_{1} q_{2}$ of elements of $\mathfrak{Q}$ is connected by $\delta$. Two classes $\mathfrak{Q}_{1}, \mathfrak{Q}_{2}$ of $\mathfrak{Q}$ are directly connected if for every $e$ there is a $q_{1 e}$ of $\mathfrak{Q}_{1}$ and a $q_{2 e}$ of $\mathfrak{Q}_{2}$ such that $\delta\left(q_{1 e} q_{2 e}\right) \leqq e$. If $\mathfrak{Q}_{2}$ is a single element $q_{2}$ and $\mathfrak{Q}_{1}, \mathfrak{Q}_{2}$ are directly connected, then $q_{2}$ must be an element of $\mathfrak{Q}_{1}$ or else a limit of a sequence of elements (not necessarily distinct) of $\mathfrak{Q}_{1}$. If two elements $q_{1}, q_{2}$ are directly connected then $\delta\left(q_{1} q_{2}\right)=0$.

\section{Systems $(\mathfrak{Q} ; \delta)$}

Theorem 1. For every coherent system $(\mathfrak{D} ; \delta)$ there is an L-equivalent coherent system $\left(\mathfrak{Q} ; \bar{\delta}^{3}\right) . \S$

In proof of this theorem $\bar{\delta}\left(q_{1} q_{2}\right)$ is defined to be $\underline{d}$, the greatest lower bound of all $d$ such that there is an element $r_{d}$ of $\mathfrak{Q}$ such that $\delta\left(q_{1} r_{d}\right) \leqq d$

* The importance of coherent systems is apparent from the fact that in such systems the derived class of every class is closed, cf. E. R. Hedrick, these Tran s a c t i o n s, vol. 12 (1911), p. 285.

† Cf. E. H. Moore, Introduction to a Form of General Analysis, § 52. New Haven Mathematical Colloquium, Yale University Press, 1910.

$\ddagger$ Cf. On the connection of an abstract set, etc., by A. D. Pitcher, A m e r i c a n J o u r n a l of $\mathrm{M}$ athematic s, vol. 36 (1914), pp. 261-266.

$\$$ An important consequence of this theorem is: In a coherent system two sequences which have a common limit have all limits in common. 
and $\delta\left(r_{d} q_{2}\right) \leqq d$. It is at once evident that $\bar{\delta}(q q)=0$ and that

$$
\bar{\delta}\left(q_{1} q_{2}\right)=\bar{\delta}\left(q_{2} q_{1}\right) \text {. }
$$

$\delta\left(q_{1} q_{2}\right) \leqq e$ implies $\bar{\delta}\left(q_{1} q_{2}\right) \leqq e$, since $r_{e}$ may be taken as $q_{1}$ or $q_{2}$. There:ore $L_{n} q_{n}=q$ implies $\bar{L}_{n} \underline{q}_{n}=q$.

We will now show that $L_{n} q_{n}=q$ implies $L_{n} q_{n}=q$. It is convenient for this purpose and for the sequel to prove the following lemma.

LemmA 1. If $L_{n} \bar{\delta}\left(q_{1 n} q_{2 n}\right)=0$ there is a sequence $\left\{r_{n}\right\}$ of elements $r_{n}$, where for any given $n, r_{n}$ may be identical with $q_{1 n}$ or $q_{2 n}$, such that

$$
L_{n} \delta\left(q_{1 n} r_{n}\right)=0 \quad \text { and } \quad L_{n} \delta\left(r_{n} q_{2 n}\right)=0 \text {. }
$$

For every $e$ there is an $n_{e}$ such that $n \geqq n_{e}$ implies $\bar{\delta}\left(q_{1 n} q_{2 n}\right) \leqq e$. From the definition of $\bar{\delta}$ there is for every $n \geqq n_{e}$ an element $r_{n e}$ such that

$$
\delta\left(q_{1 n} r_{n e}\right) \leqq e+\frac{1}{n} \quad \text { and } \quad \delta\left(r_{n e} q_{2 n}\right) \leqq e+\frac{1}{n}
$$

Let $e$ take on a sequence of values $\left\{e_{k}\right\}$ such that $L_{k} e_{k}=0$ and consider the sequence $\left\{n_{e_{k}}\right\}$ which may be taken so that $n_{e_{1}}<n_{e_{2}}<\cdots<n_{e_{k}}<n_{e_{k+1}} \cdots$. Now if $n<n_{e_{1}}, r_{n}$ may be taken at random. If $n_{k} \leqq n<n_{k+1}$, take $r_{n}$ to be $r_{n e_{k}}$. It is clear that such a sequence $\left\{r_{n}\right\}$ satisfies the given conditions.

In case $\bar{L}_{n} q_{n}=q$ we have $L_{n} \bar{\delta}\left(q_{n} q\right)=0$, and therefore by the above lemma there is a sequence $\left\{r_{n}\right\}$ such that $L_{n} \delta\left(q_{n} r_{n}\right)=0$ and $L_{n} \delta\left(r_{n} q\right)=0$. Therefore $L_{n} r_{n}=q$, and since $(\mathfrak{Q} ; \delta)$ is coherent, $L_{n} q_{n}=q$. Therefore $L_{n} q_{n}=q$ implies $L_{n} q_{n}=q$.

The system $(\mathfrak{Q} ; \bar{\delta})$ is coherent. For suppose

$$
\bar{L}_{n} q_{1 n}=q \quad \text { and } \quad L_{n} \bar{\delta}\left(q_{1 n} q_{2 n}\right)=0 .
$$

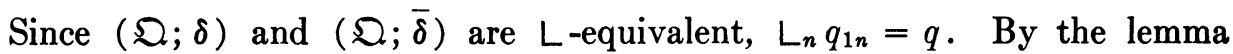
there is a sequence $\left\{r_{n}\right\}$ such that $L_{n} \delta\left(q_{1 n} r_{n}\right)=0$ and $L_{n} \delta\left(r_{n} q_{2 n}\right)=0$. Since $(\mathfrak{Q} ; \delta)$ is coherent $L_{n} r_{n}=q$ and $L_{n} q_{2 n}=q$. But $(\mathfrak{Q} ; \delta)$ and $(\mathfrak{Q} ; \bar{\delta})$ are $L$-equivalent and thus $\bar{L}_{n} q_{2 n}=q$.

The system $(\mathfrak{Q} ; \bar{\delta})$ has the property (3). For suppose $\bar{L}_{n} q_{1 n}=q=\bar{L}_{n} q_{2 n}$. Since $(\mathfrak{D} ; \delta)$ and $(\mathfrak{S} ; \bar{\delta})$ are $L$-equivalent $L_{n} q_{1 n}=q=L_{n} q_{2 n}$. Then for every $e$ there is an $n_{e}$ such that $n \geqq n_{e}$ implies $\delta\left(q_{1 n} q\right) \leqq e$ and $\delta\left(q_{2 n} q\right) \leqq e$. By definition $\bar{\delta}\left(q_{1 n} q_{2 n}\right) \leqq e$. Therefore $L_{n} \bar{\delta}\left(q_{1 n} q_{2 n}\right)=0$.

Theorem 2. Every limited system $\left(\mathfrak{Q} ; \delta^{4}\right)$ is a system $\left(\mathfrak{Q} ; \delta^{45}\right)$.

We wish to show that there is a $\phi(e)$ such that
(a) $\underset{e=0}{\operatorname{L}} \phi(e)=0$,
(b) $\delta\left(q_{1} q_{2}\right) \leqq e$,
$\delta\left(q_{2} q_{3}\right) \leqq e$

implies $\delta\left(q_{1} q_{3}\right) \leqq \phi(e)$. Given $e$, consider all possible $q_{1} q_{2} q_{3}$ such that 
$\delta\left(q_{1} q_{2}\right) \leqq e, \delta\left(q_{2} q_{3}\right) \leqq e$. Denote by $\phi(e)$ the least upper bound of all $\delta\left(q_{1} q_{3}\right)$. It is obvious that $\phi(e)$ exists, since $(\mathfrak{D} ; \delta)$ is a limited system. It is also true that $L_{e=0} \phi(e)=0$. Suppose this is not true. Then there is a positive number $a$ such that for every positive number $d$ there is an $e \leqq d$ such that $\phi(e)>a$. Thus, there is a sequence $\left\{e_{n}\right\}$ such that $L_{n} e_{n}=0$ and such that, for every $n, \phi\left(e_{n}\right)>a$. Thus there are sequences $\left\{q_{1_{n}}\right\},\left\{q_{2_{n}}\right\}$, $\left\{q_{3 n}\right\}$ such that $L_{n} \delta\left(q_{1 n} q_{2 n}\right)=0,\left\llcorner_{n} \delta\left(q_{2 n} q_{3 n}\right)=0\right.$ and yet for every $n$ $\delta\left(q_{1 n} q_{3 n}\right) \geqq a$. This contradicts the hypothesis that we have a system $\left(\mathfrak{Q} ; \delta^{4}\right)$.

Theorem 3. For every system $(\mathfrak{Q} ; \delta)$ there is an L-equivalent limited system $(\mathfrak{D} ; \bar{\delta})$.

For if

$$
\bar{\delta}\left(q_{1} q_{2}\right)=\frac{\delta\left(q_{1} q_{2}\right)}{1+\delta\left(q_{1} q_{2}\right)}
$$

$(\mathfrak{Q} ; \delta)$ and $(\mathfrak{Q} ; \bar{\delta})$ are $L$-equivalent and $\bar{\delta}\left(q_{1} q_{2}\right)$ is always less than unity. As a consequence of Theorems 2 and 3 we have the following theorem.

Theorem 4. For every system $\left(\mathfrak{D} ; \delta^{4}\right)$ there is an L-equivalent system $\left(\mathfrak{Q} ; \delta^{45}\right)$.

ThEOREM 5 . If $(\overline{\mathfrak{D}} ; \delta)$ is a compact system, a reduction of a coherent system $\left(\mathfrak{Q} ; \delta^{3}\right)$, then $(\overline{\mathfrak{Q}} ; \delta)$ is a coherent system $\left(\overline{\mathfrak{Q}} ; \delta^{34}\right)$.

$(\overline{\mathfrak{Q}} ; \delta)$ is coherent and has the property $(3)$ since it is a reduction of $(\mathfrak{Q} ; \delta)$. Suppose $(\overline{\mathfrak{D}} ; \delta)$ does not have the property $(4)$. Then there are sequences $\left\{\bar{q}_{1 n}\right\},\left\{\bar{q}_{2 n}\right\},\left\{\bar{q}_{3 n}\right\}$ such that $L_{n} \delta\left(\bar{q}_{1 n} \bar{q}_{2 n}\right)=0, L_{n} \delta\left(\bar{q}_{2 n} \bar{q}_{3 n}\right)=0$ and yet $L_{n} \delta\left(\bar{q}_{1 n} \bar{q}_{3 n}\right) \neq 0$. There are, then, sequences $\left\{\bar{q}_{1 n_{k}}\right\},\left\{\bar{q}_{3 n_{k}}\right\}$ and an $e$ such that for every $k, \delta\left(\bar{q}_{1 n_{k}} \bar{q}_{3 n_{k}}\right)>e$. Since $(\overline{\mathfrak{D}} ; \delta)$ is compact there is a sequence of $\left\{\bar{q}_{1 n_{k}}\right\}$, say $\left\{\bar{q}_{1 n_{k_{l}}}\right\}$, which has a limit $q$ (the sequence $\left\{\bar{q}_{1 n_{k_{l}}}\right\}$ may be merely $q$ repeated infinitely often). Corresponding to $\left\{\bar{q}_{1 n_{k_{l}}}\right\}$ there are sequences $\left\{\bar{q}_{2 n_{k_{l}}}\right\}$ and $\left\{\bar{q}_{3 n_{k_{l}}}\right\}$ such that $L_{l} \delta\left(\bar{q}_{1 n_{k_{l}}} \bar{q}_{2 n_{k_{l}}}\right)=0$ and $L_{l} \delta\left(\bar{q}_{2 n_{k_{l}}} \bar{q}_{3 n_{k_{l}}}\right)=0$. Therefore, since $(\mathfrak{D} ; \delta)$ is coherent, $q=L_{l} \bar{q}_{2 n_{k_{l}}}$ and $q=\left\llcorner_{l} \bar{q}_{3 n_{k_{l}}}\right.$. Then, since $\left(\mathfrak{Q} ; \delta^{3}\right)$, also $L_{l} \delta\left(\bar{q}_{1 n_{k_{l}}} \bar{q}_{3 n_{k_{l}}}\right)=0$. But this contradicts the statement that, for every $k, \delta\left(\bar{q}_{1 n_{k}} q_{3 n_{k}}\right)>e$.

Theorem 6. If $(\mathfrak{O} ; \delta)$ is a coherent system there is an L-equivalent, limited, coherent system $\left(\mathfrak{Q} ; \bar{\delta}^{3}\right)$ such that every compact reduction $(\overline{\mathfrak{Q}} ; \bar{\delta})$ of $\left(\mathfrak{O} ; \bar{\delta}^{3}\right)$ is a system $\left(\overline{\mathfrak{D}} ; \bar{\delta}^{5}\right)$.

This theorem follows at once from the successive application of Theorems $1,3,5,2$ and the fact that a reduction of a limited system is limited. It will be noted that to every compact reduction $(\overline{\mathfrak{Q}} ; \delta)$ of $(\mathfrak{Q} ; \delta)$ corresponds an $L$-equivalent compact reduction $(\overline{\mathfrak{D}} ; \bar{\delta})$ of $\left(\mathfrak{Q} ; \bar{\delta}^{3}\right)$. An important special case of the theorem occurs when $(\overline{\mathfrak{D}} ; \delta)$ is itself a compact system.

Theorem 7. If $\left(\mathfrak{D} ; \delta^{1}\right)$ is a coherent system then there is an L-equivalent, 
limited system $\left(\mathfrak{Q} ; \bar{\delta}^{13}\right)$ such that on every compact set $\overline{\mathfrak{Q}}$ of $\mathfrak{Q}, \bar{\delta}^{13}$ is a voisinage, i. e., the system $\left(\overline{\mathfrak{D}} ; \bar{\delta}^{13}\right)$ is a system $\left(\overline{\mathfrak{L}} ; \bar{\delta}^{15}\right) .^{*}$

This theorem follows from Theorem 6 and the fact that the property (1) is undisturbed by the transformations used in establishing the above theorems. $\dagger$

\section{The functions of Hahn}

Fréchet $\ddagger$ proved that if $q_{0}$ and $q_{1}$ are two elements of the set $\mathfrak{Q}$ of a system $\left(\mathfrak{Q} ; \delta^{16}\right)$ then there is a function $\mu$, continuous on the set $\mathfrak{Q}$ of the system $(\mathfrak{Q} ; \delta)$, such that $\mu\left(q_{0}\right)=0, \mu\left(q_{1}\right)=1$ and such that $q \neq q_{0}$ implies $0<\mu(q) \leqq 1$. Hahn§ proved the same theorem for a system $\left(\mathfrak{Q} ; \delta^{15}\right)$. The following is a generalization of this theorem of Fréchet and Hahn.

Theorem 8. If $\mathfrak{Q}_{0}$ and $\mathfrak{D}_{1}$ are two subsets of the set $\mathfrak{Q}$ of a coherent system $(\mathfrak{Q} ; \delta)$, which are not directly connected, then there is a function $\mu$, continuous on the set $\mathfrak{D}$ of the system $(\mathfrak{D} ; \delta)$, such that: $\mu(q)=0$ if $q$ is of $\mathfrak{Q}_{0}, \mu(q)=1$ if $q$ is of $\mathfrak{Q}_{1}$, and $0<\mu(q) \leqq 1$ if $q$ and $\mathfrak{D}_{0}$ are not directly connected. $\|$

The proof follows that of Hahn, but owing to the weaker hypotheses here employed, it seems desirable to indicate our proof.

On account of Theorem 1 we may assume that our system is a system $\left(\mathfrak{Q} ; \delta^{23}\right)$. Since $\mathfrak{\Omega}_{0}$ and $\mathfrak{Q}_{1}$ are not directly connected there is an $e_{1}$ such that if $q_{0}$ is of $\mathfrak{Q}_{0}$ and $q_{1}$ of $\mathfrak{Q}_{1}$ then $\delta\left(q_{0} q_{1}\right) \geqq e_{1}$. Denote by $\mathfrak{Q}_{2}$ the class of all $q$ 's such that for every $q_{0}, \delta\left(q_{0} q\right) \geqq e_{1} . \quad \mathfrak{Q}_{2}$ contains $\mathfrak{L}_{1} . \quad \mathfrak{\Omega}_{2}$ and $\mathfrak{Q}_{0}$ have no common limiting elements. Denote by $\mathfrak{B}$ the class of all elements which belong neither to $\mathfrak{Q}_{0}$ nor to $\mathfrak{Q}_{2}$. Thus $\mathfrak{Q}$ is divided into a sequence of three classes $\mathfrak{Q}_{0} \mathfrak{B} \mathfrak{Q}_{2}$ such that only adjacent classes may have common limiting elements and such that if $\delta\left(q^{\prime} q^{\prime \prime}\right)=0$ then $q^{\prime}, q^{\prime \prime}$ belong to the same or to adjacent classes.

A sequence $\mathfrak{Q}_{0} \mathfrak{Q}_{1} \ldots \mathfrak{Q}_{i} \mathfrak{Q}_{i+1} \ldots \mathfrak{Q}_{m}$ of classes, which is such that only adjacent classes may have limiting elements in common and such that if $\delta\left(q_{1} q_{2}\right)=0$ then $q_{1}$ and $q_{2}$ belong to the same or to adjacent classes, we call a Hahn sequence. Consider the class $\mathfrak{Q}_{i}(i \neq 0, m)$. Denote by $d_{0 q}$ the greatest lower bound of $\delta\left(q_{0} q\right)$ where $q$ is of $\mathfrak{Q}_{i}$ and $q_{0}$ ranges over $\mathfrak{Q}_{i-1}$. Denote by $d_{1 q}$ the greatest lower bound of $\delta\left(q_{1} q\right)$ where $q_{1}$ ranges over $\mathfrak{Q}_{i+1}$.

* The following is an example of a system $(\mathfrak{Q} ; \delta)$ which is of interest here:

$\mathfrak{Q} \equiv$ all real numbers $x$ such that $0 \leqq x \leqq 1 ; \quad \delta\left(x_{1} x_{2}\right) \equiv\left|x_{1}-x_{2}\right| \quad x_{1}<1, \quad x_{2}<1$;

$$
\delta(01) \equiv \delta(10) \equiv 1 \text {; }
$$

$$
\delta(1 x) \equiv \delta(x 1) \equiv 1-x \quad x \geqq \frac{1}{2} ; \quad \delta(1 x) \equiv 1 / x \quad 0<x<\frac{1}{2} .
$$

The system $(\mathfrak{Q} ; \delta$ ) is extremal, coherent, perfect, but not limited.

† From Theorem 7 and the work of Chittenden (loc. cit.) it follows that in compact sets coherence and écart are infinitesimally equivalent.

$\ddagger$ Loc. cit., $\S 51$.

$\S$ Monatshefte für Mathematik und Physik, vol. 19 (1908), pp. 251.

$\|$ It is obvious that $\mu(q)=0$ if $\mathfrak{Q}_{0}$ and $q$ are directly connected. 
If $d_{0 q} \leqq d_{1 q}$ assign $q$ to a class $\mathfrak{Q}_{i 0}$. If $d_{0 q}>d_{1 q}$ assign $q$ to a class $\mathfrak{Q}_{i 1}$. Denote by $D$ this principle of division.

Lемма 2. The principle of division D transform's a Hahn sequence into a Hahn sequence.

In proof of this we must show that in the new sequence of classes only adjacent classes may have common limiting elements. It is clear that we need only show that $\mathfrak{Q}_{i 0}$ and $\mathfrak{Q}_{i+1}$ have no common limiting elements and that $\mathfrak{Q}_{i 1}$ and $\mathfrak{Q}_{i-1}$ have no common limiting elements. Suppose $\mathfrak{Q}_{i 0}$ and $\mathfrak{Q}_{i+1}$ have a common limiting element $q$. Then there is a sequence $\left\{q_{0_{n}}\right\}$ of $\mathfrak{Q}_{i 0}$ and a sequence $\left\{q_{1 n}\right\}$ of $\mathfrak{Q}_{i+1}$ which have $q$ for a limit. Then from $\delta^{3}$ it follows that $L_{n} \delta\left(q_{0 n} q_{1 n}\right)=0$ and from the way the division of $\mathfrak{Q}_{i}$ was effected there must be a sequence $\left\{q_{n}\right\}$ of $\mathfrak{Q}_{i-1}$ such that $L_{n} \delta\left(q_{n} q_{0 n}\right)=0$. Therefore $L_{n} q_{n}=q$ and $\mathfrak{Q}_{i-1}, \mathfrak{Q}_{i+1}$ have a common limiting element. This however contradicts the fact that the original sequence $\mathfrak{Q}_{0} \ldots \mathfrak{Q}_{i} \ldots \mathfrak{Q}_{m}$ is a Hahn sequence. Similarly we may prove that $\mathfrak{Q}_{i 1}$ and $\mathfrak{Q}_{i-1}$ have no common limiting elements.

We must also see that if $\delta\left(q_{1} q_{2}\right)=0$ then $q_{1}$ and $q_{2}$ belong to the same or to adjacent classes. It is obvious that we need only see that $\mathfrak{Q}_{i 0}$ and $\mathfrak{Q}_{i+1}$ can not contain elements $q_{i 0}$ and $q_{i+1}$ respectively such that $\delta\left(q_{i 0} q_{i+1}\right)=0$ and that $\mathfrak{Q}_{i 1}$ and $Q_{2-1}$ can not contain elements $q_{i 1}$ and $q_{i-1}$ respectively such that $\delta\left(q_{i 1} q_{i-1}\right)=0$. Suppose $\delta\left(q_{i 0} q_{i+1}\right)=0$ and that $q_{i 0}$ is of $\mathfrak{Q}_{i 0}$ and $q_{\text {ot1 }}$ of $\mathfrak{Q}_{\imath+1}$. Then, from the way the division is effected, $\mathfrak{Q}_{\imath-1}$ must contain an element $q_{i-1}$ such that $\delta\left(q_{i-1} q_{i 0}\right)=0$. Then, since we have a coherent system, $\delta\left(q_{i-1} q_{i+1}\right)=0$, which again contradicts the hypothesis that the original sequence is a Hahn sequence. Similarly for $\mathfrak{Q}_{i 1}$ and $\mathfrak{Q}_{i-1}$. Thus the lemma is proved.

Now the above sequence $\mathfrak{Q}_{0} \mathfrak{P} \mathfrak{Q}_{2}$ is a Hahn sequence and by use of the principle of division $D$ a succession of Hahn sequences may be formed of which the first one is

$$
\mathfrak{Q}_{0} \mathfrak{P}_{0} \mathfrak{P}_{1} \mathfrak{Q}_{2} \text {, }
$$

and the second one,

$$
\mathfrak{Q}_{0} \mathfrak{P}_{00} \mathfrak{P}_{01} \mathfrak{P}_{10} \mathfrak{P}_{11} \mathfrak{Q}_{2},
$$

and the $k$ th one,

$$
\mathfrak{Q}_{0} \mathfrak{P}_{00} \ldots \mathfrak{0 0}_{00} \mathfrak{P}_{00} \ldots \mathfrak{P}_{i_{1} i_{2}} \ldots i_{k} \ldots \mathfrak{P}_{11} \ldots 10 \mathfrak{P}_{11} \ldots 11 \mathfrak{Q}_{2} .
$$

It is not difficult to show that if $q$ is in a class $\mathfrak{D}_{i_{1} i_{2}} \ldots i_{k}$ and if $\left\{q_{n}\right\}$ is a sequence of elements, distinct or not, such that $L_{n} q_{n}=q$ then there is an $n_{k}$ and a class adjacent to $\mathfrak{Q}_{i_{1} i_{2}} \ldots i_{k}$ such that for $n \geqq n_{k}, q_{n}$ belongs to $\mathfrak{Q}_{i_{1} i_{2}} \ldots i_{k}$ or to this adjacent class. ${ }^{*}$ Otherwise the fact that at every stage the sequence of classes is a Hahn sequence is contradicted.

Thus to each element $q$ not of $\mathfrak{Q}_{0}$ nor of $\mathfrak{Q}_{2}$ is associated an infinite sequence

\footnotetext{
* It is understood here that the class $\mathfrak{Q}_{i_{1}} i_{2} \ldots i_{k}$ may be the class $\mathfrak{Q}_{0}$ or $\mathfrak{Q}_{2}$.
} 
of numbers,

$$
i_{1}, i_{2}, i_{3}, \cdots, i_{k}, \cdots \quad\left(i_{k}=0 \text { or } 1\right),
$$

the first $k$ of which are identical with the subscript to the class of stage $k$ to which $q$ belongs. Define a function $\mu_{1}$ as follows: $\mu_{1}(q)=0$ if $q$ is of $\mathfrak{Q}_{0}$; $\mu_{1}(q)=1$ if $q$ is of $\mathfrak{Q}_{2}$;

$$
\mu_{1}(q)=\frac{i_{1}}{2}+\frac{i_{2}}{2^{2}}+\frac{i_{3}}{2^{3}}+\cdots+\frac{i_{k}}{2^{k}}+\cdots,
$$

if $q$ is of $\mathfrak{B}$. That $\mu_{1}$ is continuous is readily seen from the foregoing and from the fact that if $q_{1}$ and $q_{2}$ are of adjacent classes of stage $k$ then

$$
\left|\mu_{1}\left(q_{1}\right)-\mu_{2}\left(q_{2}\right)\right| \leqq \frac{1}{2^{k-1}} .
$$

Now consider a sequence $\left\{e_{n}\right\}$ of numbers $e_{n}$, of which $e_{1}$ is used above, such that for every $n, e_{n-1}<e_{n}<e_{n+1}$ and such that $L_{n} e_{n}=0$. Corresponding to $e_{1}$ we have $\mu_{1}$, corresponding to $e_{2}$ we have $\mu_{2}$, etc. The function $\mu=\sum_{n=1}^{n=\infty} \mu_{n} / 2^{n}$ is a continuous function $\mu$ having the desired properties, viz., $\mu(q)=0$ if $q$ is of $\mathfrak{Q}_{0}, \mu(q)=1$ if $q$ is of $\mathfrak{Q}_{2}$, and $0<\mu(q) \leqq 1$ if $\mathfrak{Q}_{0}$ and $q$ are not directly connected. Obviously $\mathfrak{Q}_{0}$ and $\mathfrak{Q}_{1}$ may be classes each consisting of a single element.

Theorem 9. A necessary and sufficient condition that every continuous function on a class $\overline{\mathfrak{Q}}$ of a class $\mathfrak{Q}$ of a coherent system $(\mathfrak{Q} ; \delta)$ be bounded and assume its bounds is that $\overline{\mathfrak{Q}}$ be self-compact.

That the condition is sufficient is easily proved. The necessity of the condition may be proved after the manner of Hahn.* The reader who has grasped the significance of the condition (2) on $\delta$ as exemplified above will have no difficulty in carrying out the proof of Hahn under these milder hypotheses.

\section{Biextremal connected systems $(\mathfrak{Q} ; \delta)$}

A system $(\mathfrak{Q} ; \delta)$ is said to be biextremal in case it is true that when two sequences $\left\{q_{1 n}\right\},\left\{q_{2 n}\right\}$ are such that $L_{n} \delta\left(q_{1 n} q_{2 n}\right)=0$ then there is a pair of sequences $\left\{q_{1 n_{k}}\right\},\left\{q_{2 n_{k}}\right\}$ (subsequences of $\left\{q_{1 n}\right\},\left\{q_{2 n}\right\}$ respectively) which have a common limit.

ThEoREM 10. Every biextremal system $(\mathfrak{Q} ; \delta)$ is also extremal.

For consider sequence $\left\{q_{n}\right\}$ of distinct elements. Then $L_{n} \delta\left(q_{n} q_{n}\right)=0$ and there is a sequence $q_{n_{k}}$ which has a limit $q$.

Theorem 11. If a function $\mu$ is continuous on a set $\mathfrak{O}$ of a biextremal system $(\mathfrak{Q} ; \delta)$, then $\mu$ is uniformly continuous.

For suppose $\mu$ were not uniformly continuous. Then there must be an $e$

* Loc. cit. 
such that for every $n$ there is an element $q_{1_{n}}$ and an element $q_{2_{n}}$ such that $\delta\left(q_{1 n} q_{2 n}\right)<1 / n$ and yet $\left|\mu\left(q_{1 n}\right)-\mu\left(q_{2 n}\right)\right| \geqq e$. Now $L_{n} \delta\left(q_{1 n} q_{2 n}\right)=0$ and since $(\mathfrak{O} ; \delta)$ is biextremal, there is a pair of sequences $\left\{q_{1 n_{k}}\right\},\left\{q_{2 n_{k}}\right\}$ which have a common limit, say $q_{0}$. The continuity of $\mu$ at $q_{0}$ affords a contradiction.

THEOREM 12. If a function $\mu$ is continuous on the set $\mathfrak{Q}$ of a biextremal, connected system $(\mathfrak{D} ; \delta)$, then $\mu$ is uniformly continuous, bounded, assumes its bounds and every value between these bounds.

This theorem follows from Theorems 10 and 11 and Fréchet, ${ }^{*}$ page 8, corollary, and the paper by Pitcher already cited, page 264, Theorem III.

Thus every continuous function on a set $\mathfrak{Q}$ of a biextremal connected system $(\mathfrak{Q} ; \delta)$ possesses the important properties which we usually associate with a continuous function on a closed interval or region. However in a particular case there may be very few such continuous functions. Indeed it may happen that only constant functions are continuous. For example let $\mathfrak{Q}$ be any set of elements and for every $q_{1}, q_{2}$ let $\delta\left(q_{1} q_{2}\right)=0$. The system thus defined is biextremal and connected. However every function continuous on the set $\mathfrak{Q}$ of $(\mathfrak{Q} ; \delta)$ is constant. Certainly a proper set of continuous functions on a set $\mathfrak{Q}$ should, in general, contain functions other than the constant functions.

\section{Systems ( $\mathfrak{O} ; \delta$ ) ADMitTing a UNIFORMLY PROPER ClASS OF CONTINUOUS FUNCTIONS}

We will say that the class of all continuous functions on the set $\mathfrak{Q}$ of a system $(\mathfrak{Q} ; \delta)$ is a uniformly proper class in case the following conditions are satisfied.

(A) Every continuous function on $\mathfrak{Q}$ is bounded and assumes its bounds.

(B) Every continuous function on $\mathfrak{Q}$ is uniformly continuous.

$(C)$ Every continuous function on $\mathfrak{O}$ assumes every value between each pair of its values.

(D) If $\mathfrak{Q}_{0}$ and $\mathfrak{Q}_{1}$ are two classes of $\mathfrak{Q}$ which are not directly connected, there is a function $\mu$, continuous on the set $\mathfrak{Q}$ of the system $(\mathfrak{Q} ; \delta)$ such that $\mu(q)=0$ if $q$ is of $\mathfrak{Q}_{0}, \mu(q)=1$ if $q$ is of $\mathfrak{Q}_{1}$, and $0<\mu(q) \leqq 1$ if $q$ and $\mathfrak{Q}_{0}$ are not directly connected.

(E) If $q_{1} \neq q_{2}$ there is a continuous function $\mu$ such that $\mu\left(q_{1}\right) \neq \mu\left(q_{2}\right)$.

In the sequel we give a set of conditions on $(\mathfrak{Q} ; \delta)$ which are necessary and sufficient that the class of all continuous functions on $\mathfrak{Q}$ be a uniformly proper class. This set of conditions thus gives rise to the uniformly proper theory of continuous functions mentioned early in this paper.

A system $(\mathfrak{D} ; \delta)$ is said to be $L$-unique in case no sequence $\left\{q_{n}\right\}$ has more than one limit $q$.

* Loc. cit. 
Theorem 13. A biextremal, L-unique system $(\mathfrak{D} ; \delta)$ is a coherent system.

For suppose $q=\mathrm{L}_{n} q_{1 n}$ and $\mathrm{L}_{n} \delta\left(q_{1 n} q_{2 n}\right)=0$ and $q \neq \mathrm{L}_{n} q_{2 n}$. Then there is an $e$ and a subsequence $\left\{q_{2 n_{k}}\right\}$ of $\left\{q_{2 n}\right\}$ such that for every $k, \delta\left(q_{2 n_{k}} q\right)>e$. But $L_{k} \delta\left(q_{1 n_{k}} q_{2 n_{k}}\right)=0$ and, since $(\mathfrak{Q} ; \delta)$ is biextremal, there must be sequences $\left\{q_{1 n_{k_{l}}}\right\}$ and $\left\{q_{2 n_{k_{l}}}\right\}$ of $\left\{q_{1 n_{k}}\right\},\left\{q_{2 n_{k}}\right\}$ respectively which have a common limit. Since $(\mathfrak{Q} ; \delta)$ is $L$-unique this limit must be $q$. Thus we have a contradiction.

Theorem 14. The class of all continuous functions on the set $\mathfrak{Q}$ of a biextremal, connected, L-unique system $(\mathfrak{D} ; \delta)$ is a uniformly proper class.

This theorem is a consequence of Theorems 13, 12, and 8 . In applying Theorem 8 we should note that if a system $(\mathfrak{D} ; \delta)$ is $L$-unique then $\delta\left(q_{1} q_{2}\right)$ $=0$ implies $q_{1}=q_{2}$ and no pair of classes each consisting of a single element can be directly connected.

Theorem 15. If a system $(\mathfrak{Q} ; \delta)$ has the property $E$ then $(\mathfrak{Q} ; \delta)$ is Lunique.

For if there be a sequence with two limits $q_{1}$ and $q_{2}$ and if $\mu$ is a continuous function then $\mu\left(q_{1}\right)=\mu\left(q_{2}\right)$.

TheoRem 16. If a system $(\mathfrak{Q} ; \delta)$ has the properties $A, D, E$ then $(\mathfrak{Q} ; \delta)$ is an extremal system.

By the previous theorem $(\mathfrak{Q} ; \delta)$ is an L-unique system. Suppose $(\mathfrak{Q} ; \delta)$ were not an extremal system. Then there is a sequence $\left\{q_{n}\right\}$ of distinct elements with no limiting elements. Divide $\left\{q_{n}\right\}$ into two classes:

$$
\mathfrak{Q}_{0}=q_{j+1}, q_{j+2}, q_{j+3}, \cdots, q_{j+k}, \cdots ; \quad \mathfrak{Q}_{1}=q_{1}, q_{2}, \cdots, q_{j} .
$$

Each of these classes is closed, they are not directly connected, and, since we have $L$-unique, neither class is directly connected with any element not in the class. Then, by the condition $D$, there is a continuous function $\mu_{j}$ such that $\mu_{j}\left(q_{i}\right)=1,(i \leqq j) ; \mu_{j}\left(q_{i}\right)=0(i>j) ; 0<\mu_{j}(q) \leqq 1$ for $q$ not of $\left\{q_{n}\right\}$.

Now consider the function $\mu=\sum_{j=1}^{j=\infty} \mu_{j} / 2^{j}$, which is nowhere 0 , and which is such that $\mu\left(q_{1}\right)=1, \mu\left(q_{2}\right)=\frac{1}{2}, \cdots, \mu\left(q_{j}\right)=1 / 2^{j-1}, \cdots$. Here $1 / \mu$ is well defined, continuous, but not bounded. This contradicts the hypothesis that $(\mathfrak{Q} ; \delta)$ is a system with the property $A$.

Theorem 17. If a system $(\mathfrak{Q} ; \delta)$ has the property $C$ then $(\mathfrak{D} ; \delta)$ is a connected system.

This theorem is proved in the paper by Pitcher referred to above, page 265, Theorem $\mathrm{Vb}$.

Theorem 18. If a system $(\mathfrak{Q} ; \delta)$ has the properties $A, B, D, E$, then $(\mathfrak{O} ; \delta)$ is biextremal.

For suppose $(\mathfrak{Q} ; \delta)$ is not biextremal. Then there is a pair of sequences $\left\{q_{1 n}\right\},\left\{q_{2 n}\right\}$ such that $L_{n} \delta\left(q_{1 n} q_{2 n}\right)=0$ but such that no sequences $\left\{q_{1 n_{k}}\right\}$, 
$\left\{q_{2 n_{k}}\right\}$ have a common limit. We may suppose that neither $\left\{q_{1 n}\right\}$ nor $\left\{q_{2 n}\right\}$ contains a single element repeated infinitely often. For suppose $\left\{q_{1 n}\right\}$ contains a single element $q$ repeated infinitely often. Then this element $q$ forms an identical sequence $\left\{q_{1 n_{k}}\right\}, q_{1 n_{k}}=q$ for every $k$. Moreover the sequences $\left\{q_{1 n_{k}}\right\}$ and $\left\{q_{2 n_{k}}\right\}$ have a common limit, the element $q$ itself. This contradicts the statement that no sequences $\left\{q_{1 n_{k}}\right\},\left\{q_{2 n_{k}}\right\}$ have a common limit. By Theorem 16 , the system $(\mathfrak{Q} ; \delta)$ is an extremal system. Then there is a sequence $\left\{q_{1 n_{k}}\right\}$, a subsequence of $\left\{q_{1 n}\right\}$, which has a limit $q_{1}$. The sequence $\left\{q_{2 n_{k}}\right\}$ contains an infinity of distinct elements and, since $(\mathfrak{Q} ; \delta)$ is extremal, there is a subsequence $\left\{q_{2 n_{k}}\right\}$ of $\left\{q_{2 n_{k}}\right\}$ which has a limit $q_{2}$. Thus

$$
\left\llcorner\delta\left(q_{1 n_{k_{l}}} q_{2 n_{k_{l}}}\right)=0, \quad L_{l} q_{1 n_{k_{l}}}=q_{1}, \quad L_{l} q_{2 n_{k_{l}}}=q_{2} .\right.
$$

By Condition $B$ a function $\mu$, continuous on $\mathfrak{Q}$, is uniformly continuous. Therefore $L_{l}\left|\mu\left(q_{1 n_{k_{l}}}\right)-\mu\left(q_{2 n_{k_{l}}}\right)\right|=0$. Also $L_{l} \mu\left(q_{1 n_{k_{l}}}\right)=\mu\left(q_{1}\right)$ and $L_{l} \mu\left(q_{2 n_{k_{l}}}\right)=\mu\left(q_{2}\right)$. Thus if $\mu$ is continuous $\mu\left(q_{1}\right)=\mu\left(q_{2}\right)$. But this contradicts the hypothesis that $(\mathfrak{Q} ; \delta)$ has the property $E$.

From Theorems 14, 15, 17, 18 we have the following theorem.

THEOREM 19. A necessary and sufficient condition that the class of all continuous functions on the set $\mathfrak{Q}$ of a system $(\mathfrak{Q} ; \delta)$ be a uniformly proper class is that $(\mathfrak{Q} ; \delta)$ be biextremal, connected, L-unique.

The significance of this theorem is emphasized by Theorem 7 and the theorem of Chittenden, previously referred to, that for every system $\left(\mathfrak{Q} ; \delta^{15}\right)$ there is an $L$-equivalent system $\left(\mathfrak{Q} ; \bar{\delta}^{16}\right)$.

\section{INDEPENDENCE CONSIDERATIONS}

The notion of the complete independence of a set of properties or postulates has been introduced into the literature by $\mathrm{E}$. H. Moore.* We give here a set of eight examples of systems $(\mathfrak{Q} ; \delta)$. Each example is preceded by a combination of plus and minus signs which indicate the character of the example as to the properties biextremal, connected, L-unique. Thus $(+-+)$ denotes that the following system $(\mathfrak{Q} ; \delta)$ is biextremal, not connected, L-unique.

$(+++) \mathfrak{Q} \equiv$ all real numbers $x$ such that $0 \leqq x \leqq 1$.

$$
\delta\left(x_{1} x_{2}\right)=\left|x_{1}-x_{2}\right| \text {. }
$$

$(-++) \mathfrak{Q} \equiv$ all real numbers $x$ such that $0<x \leqq 1$.

$$
\delta\left(x_{1} x_{2}\right)=\left|x_{1}-x_{2}\right| \text {. }
$$

$(+-+) \mathfrak{Q} \equiv$ all real numbers $x$ such that $0 \leqq x \leqq 1$ or $2 \leqq x \leqq 3$.

* Loc. cit.

$$
\delta\left(x_{1} x_{2}\right)=\left|x_{1}-x_{2}\right|
$$


$(++-) \mathfrak{Q} \equiv$ all real numbers $x$ such that $0 \leqq x \leqq 1$ and an element $*$.

$$
\delta\left(x_{1} x_{2}\right)=\left|x_{1}-x_{2}\right| ; \quad \delta(x *)=|x-1| .
$$

$(--+) \mathfrak{Q} \equiv$ all real numbers $x$ such that $0<x \leqq 1$ or $2 \leqq x \leqq 3$.

$$
\delta\left(x_{1} x_{2}\right)=\left|x_{1}-x_{2}\right| \text {. }
$$

$(+--) \mathfrak{Q} \equiv$ all real numbers $x$ such that $0 \leqq x \leqq 1$ or $2 \leqq x \leqq 3$ and an element $* . \quad \delta\left(x_{1} x_{2}\right)=\left|x_{1}-x_{2}\right| ; \delta(x *)=|x-1|$.

$(-+-) \mathfrak{Q} \equiv$ all real numbers $x$ such that $0<x \leqq 1$ and an element $*$.

$$
\delta\left(x_{1} x_{2}\right)=\left|x_{1}-x_{2}\right|, \quad \delta(x *)=|x-1| .
$$

$(---) \mathfrak{Q} \equiv$ all real numbers $x$ such that $0<x \leqq 1$ or $2 \leqq x \leqq 3$ and an element $* . \quad \delta\left(x_{1} x_{2}\right)=\left|x_{1}-x_{2}\right| ; \delta(x *)=|x-1|$.

From these eight examples we have the following theorem.

Theorem 20. The properties biextremal, connected, L-unique of systems $(\mathfrak{Q} ; \delta)$ are completely independent.

\section{ANalysis of the properties biextremal and COHERENT}

We have already seen that every biextremal system $(\mathfrak{Q} ; \delta)$ is extremal and that every biextremal, $L$-unique system $(\mathfrak{Q} ; \delta)$ is coherent. It is also true that every coherent, extremal system $(\mathfrak{Q} ; \delta)$ is biextremal. For suppose we have a coherent, extremal system $(\mathfrak{O} ; \delta)$ and two sequences $\left\{q_{1 n}\right\},\left\{q_{2 n}\right\}$ such that $L_{n} \delta\left(q_{1 n} q_{2 n}\right)=0$. We wish to show that there is a pair of sequences $\left\{q_{1 n_{k}}\right\}\left\{q_{2 n_{k}}\right\}$ which have a common limit. If either $\left\{q_{1 n}\right\}$ or $\left\{q_{2 n}\right\}$ contain a single element repeated infinitely often our contention is established at once. If such is not the case, then, since $(\mathfrak{Q} ; \delta)$ is extremal, there is a sequence $\left\{q_{1 n_{k}}\right\}$ which has a limit $q$. But since $(\mathfrak{Q} ; \delta)$ is coherent and

we have also $L_{k} q_{2 n_{k}}=q$.

$$
\left\llcorner_{k} \delta\left(q_{1 n_{k}} q_{2 n_{k}}\right)=0\right. \text {, }
$$

Theorem 21. For L-unique systems $(\mathfrak{Q} ; \delta)$ the property biextremal is equivalent to the two properties extremal and coherent.

Thus the four properties: extremal, connected, $L$-unique, coherent serve equally well as the basis of a uniformly proper theory of continuous functions and in fact are both necessary and sufficient for the same. The reader can show, without serious difficulty, that these four properties are also completely independent.

The following properties are interesting and important.

(a) Every sequence $\left\{q_{n}\right\}$ contains a sequence $\left\{q_{n k}\right\}$ which has a limit.

(b) If a sequence $\left\{q_{n}\right\}$ has a limit and $L_{n} \delta\left(q_{1 n} q_{2 n}\right)=0$, then $\left\{q_{1 n}\right\}$ and $\left\{q_{2 n}\right\}$ have subsequences $\left\{q_{1 n_{k}}\right\},\left\{q_{2 n_{k}}\right\}$ which have a common limit. 
(c) If $q$ is a limit of a sequence $\left\{q_{1 n}\right\}$ which has a limit in common with a sequence $\left\{q_{2 n}\right\}$ then $q$ is a limit of $\left\{q_{2 n}\right\}$.

Theorem 22. The property biextremal is equivalent to properties $(a)$ and $(b)$ and the property coherent is equivalent to properties $(b)$ and $(c)$.

The first part of the theorem is readily proved. It is also not difficult to see that every coherent system possesses the Property $(b)$. To see that every coherent system possesses the Property (c) note that if $q_{1}=L_{n} q_{1 n}$ and $L_{n} q_{1 n}=q_{0}=L_{n} q_{2 n}$ then $\delta\left(q_{1} q_{0}\right)=0$ and $q_{1}=L_{n} q_{2 n}$. Also if $(\mathfrak{Q} ; \delta)$ has the Properties (b), (c) and if $L_{n} q_{1 n}=q$ and $L_{n} \delta\left(q_{1 n} q_{2 n}\right)=0$ then there is an element $q_{0}$ and sequences $\left\{q_{1 n_{k}}\right\}\left\{q_{2 n_{k}}\right\}$ such that $L_{k} q_{1 n_{k}}=q_{0}=L_{k} q_{2 n_{k}}$. Then from (c) $q$ is a limit of $\left\{q_{2 n_{k}}\right\}$. The supposition that $q$ is not a limit of $\left\{q_{2 n}\right\}$ evidently leads to a contradiction. Thus properties $(b)$ and (c) imply the property coherent. This theorem focuses on the importance of the properties (b) and (c) and suggests sets of basic properties other than those given above. Western Reserve University, Cleveland, Оhio

University of Illinois, Champaign, Illinois 